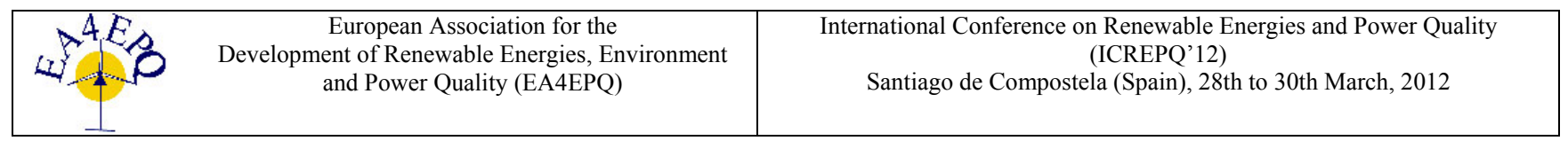

\title{
Analysis of the reconstruction of load curves in an urban Low Voltage substation
}

\author{
M. Mendoza ${ }^{1}$, P. Zorzano ${ }^{1}$, A. Falces ${ }^{1}$, E. Tarancón ${ }^{2}$, E. Zorzano ${ }^{1}$ and P. Lara
}

\author{
${ }^{1}$ Department of Electrical Engineering \\ ${ }^{2}$ Department of Mecanical Engineering \\ E.T.S.I.I., University of La Rioja \\ C/ Luis de Ulloa 20, 26004 Logroño (Spain)
}

Phone number:+34 941299491, e-mail: pedro.lara@unirioja.es

\begin{abstract}
The study of the load curves in an urban Low Voltage (LV) substation is a work that has been carried out over several years.
\end{abstract}

A load curve is a sequence of values, associated with the energy consumption, in time. This sequence can be considered as a signal and, in order to study it the techniques and tools of signal processing can be applied.

This article applies different signal processing tools to analyze the interpolation of the load curve of an urban LV substation. The above mentioned interpolation can be necessary for both the case of lost measurements reconstruction and for the estimation of non-existent measurements.

The tests have been carried out on a records database of an actual LV substation. The power records have been made over more than 10 years. This substation has been selected because its consumptions were representative of the entire city, not only of the influence area. This choice has been made comparing LV substation measurements with the sum of the measurements of the high voltage lines that supply electricity to the city.

The extracted conclusions are valid in trying to improve future predictive models of lost measurements reconstruction, data construction, etc.

\section{Key words}

Load curve, urban electric power demand, curves interpolation, LV substation.

\section{Introduction}

The load curve is a graph used both by engineers and electric power producers to see/show how consumers use this power during a given period of time. Actually, the data associated with the load curve is one of the sets of most valuable measurements of electrical companies.
The study of load curves is useful for creating energy models [1]. These models are used both for the planning and operation of the power systems and for the determination of the electrical power costs [2].

Identifying load curves even makes it possible to reduce the maximum peaks of consumption [3] and be able to operate more efficiently. It is also possible to make customer classifications [4], [5], [7], which are useful in processes such as load forecasting [6].

The identification and prediction of the load curve is not only a necessity for electrical power generation and distribution companies, but can also be useful for electric power businesses that operate as intermediaries (packets of energy purchased from the large companies in the liberalized market and subsequently sold to small and medium sized customers unable to operate in this market).

The load curves are also studied to allocate energy losses in the power system transmission and distribution for their subsequent use in the calculation of electricity prices.

In any event, the collection of data is a costly task, taking into account the time and volume of data involved, and one which has to be carried out continuously. For this reason it is important to also consider the best way of making data compressions and of handling the lack of data in some period of time. This missing data can be interpolated or considered as false [8].

This article analyzes the best way to interpolate values of a load curve of an urban LV substation. To be able to perform reliable estimates, the records made in a LV substation of the town of Logroño have been used.

The LV substation is located near the centre of Logroño, a medium sized town in the north of Spain, with a population of nearly 160000 inhabitants. This LV substation supplies electric power to almost 450 consumers. Among these there are customers of the third sector (hostelry, shops and other services); in addition, 
there are consumers from several blocks of flats corresponding to people of different social groups. That is to say, the studied LV substation shows a representative consumption of the whole town, which has been considered as a group.

\section{Methodology}

The methodology used to determine the best model of interpolation is very simple. Basically it can be summarized in three steps. In the first place the load curves are reconstructed implementing the different object of study algorithms. The second step consists in comparing the waveforms obtained in the first step with the original waveform, evaluating the parameters of error, as well as the position where they have the maximum. The third and last step is to make a comparison of the results obtained in step 2 for each of the applied algorithms and extract conclusions.

\section{A. Reconstruction of the load curves.}

Actually this step involves several different stages. Initial count with a database of actual records, sampled each minute, the load curve of an urban LV substation of Logroño. This substation has been the object of measuring for more than 10 years. Currently measurements are still being taken.

On the above mentioned database, load curves are separated into sections of 1 day in length (1440 minutes / records). These sections will be used to test the object of study methods of interpolation.

The interpolation can be done using two large groups of tools: the holders and the orthogonal decompositions.

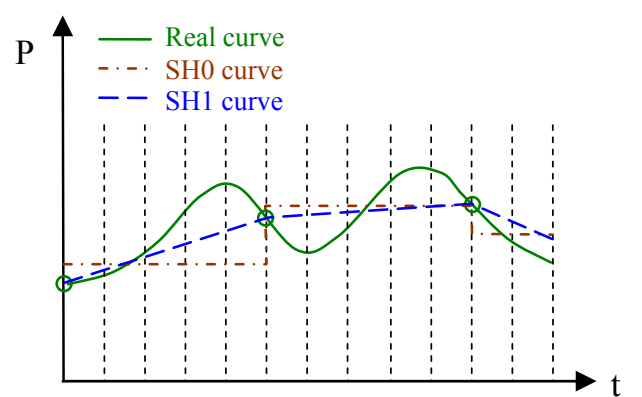

Fig. 1. Window of curve and holder technique used to interpolate

The holders, $\mathrm{SH}$, calculated the point of interpolation according to the values of the nearest points to which one wants to calculate. The most commonly used are those of order 0 and 1. Another characteristic is that the computational effort is very low. Figure 1 graphically displays the process of interpolation for $\mathrm{SH} 0$ and $\mathrm{SH} 1$.

When the information is interpolated using an orthogonal decomposition, all the points on the curve, not only the next, are used. In the first place the signal analysis allows the signal to be adjusted to a mathematical equation that runs through all of the points used. Subsequently this equation is evaluated at the points of interest to us. The idea is represented graphically in figure 2 .
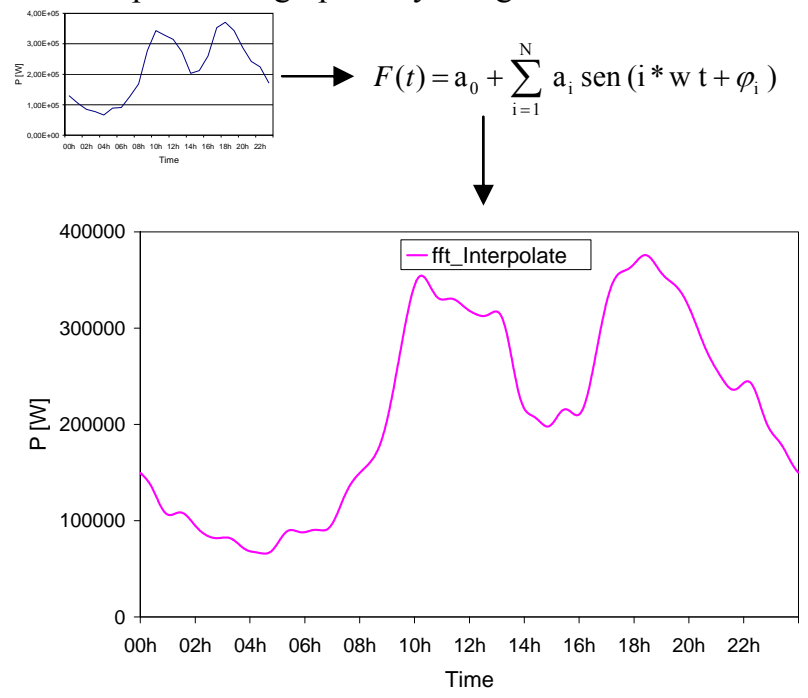

Fig. 2. Interpolation process based on FFT decomposition.

The FFT and wavelets are among the most popular orthogonal decompositions. Implementing the FFT is really involves translating a signal from the time domain to the frequency. Applying wavelet transform involves translating a domain that centres neither on the time nor on the frequency; in addition, each mother wavelet implies a different decomposition.

\section{B. Comparison of the original and the interpolated waveforms}

The comparison of the waveforms, figure 3 , is done point by point. For each of the minutes of the day, the standardized absolute and the relative error are computed (1).

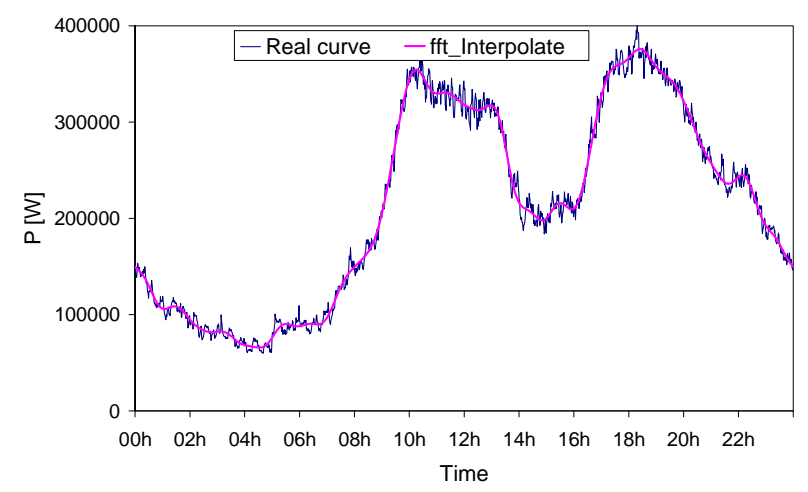

Fig. 3. Load curve of one day and interpolated waveform, calculated using FFT technique with 24 harmonics to interpolate every minute.

$$
\text { Error }_{\text {Rel }} \%=\frac{\text { real value }- \text { interpolated value }}{\text { real value }} * 100
$$

After calculating the relative and absolute errors throughout the curve, the minute in which the maximum of both errors takes place is identified.

Furthermore, both the average value (2), and the effective value of the error are evaluated (3) for the whole curve. 


$$
\begin{aligned}
& \text { Error }_{\mathrm{AVG}}=\frac{\sum_{\mathrm{i}=1}^{\mathrm{N}} \text { Absolut error }_{\mathrm{i}}}{\mathrm{N}} \\
& \text { Error }_{\text {RMS }}=\sqrt{\frac{\sum_{i=1}^{\mathrm{N}} \text { Absolut error }_{i}^{2}}{\mathrm{~N}}}
\end{aligned}
$$

These errors are also calculated as standardized in order to compare values in a more coherent manner.

The process of comparing curves and evaluation of errors is repeated for each of the interpolations made in all the curves drawn from the database.

\section{Comparison of results.}

In the comparison, factors such as: which of the methods used commits minor errors, the position of the values of maximum error, the variation of an error depending on a parameter of interpolation and others are evaluated.

To make the comparison, homogeneous groups of waveforms must first be established. It is believed that two load curves, interpolated, belong to a group of comparison when the same number of intermediate points is evaluated or when one of its parameters for a model of interpolation given is changed (figure 4 shows the error of interpolation based on FFT according to the number of harmonics used).

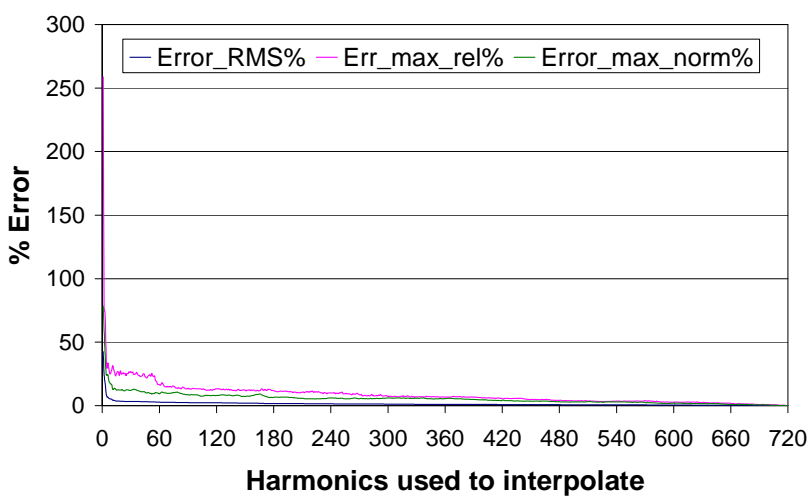

Fig. 4. Error $_{\text {RMS }}$ of a load curve as a function of the harmonics used to compute the curve every minute in a Tuesday of winter.

\section{Results}

The study of the comparisons among the different models of interpolation is performed from different points of view. Next some of the results are set out.

When a reconstruction is made by means of holders $\mathrm{SH} 0$, the error RMS grows in an approximate way to a logarithmic curve, though the function of error that best adjusts is polynomial (as shown in figure 5).

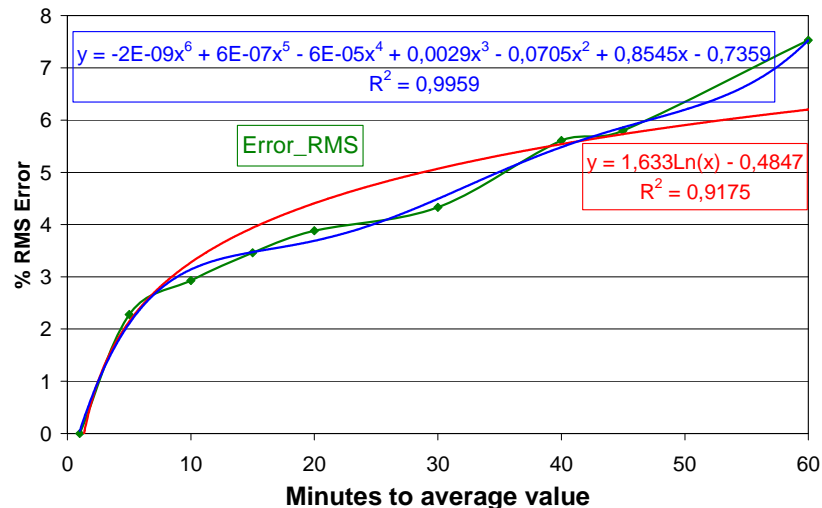

Fig. 5. Error $_{\text {RMS }}$ of a load curve as a function of minutes used to extend by means of a $\mathrm{SHO}$ in a Tuesday of winter.

Another result that can be deduced from the study of the errors committed on having extended the curve by means of SH0, is that the maximum errors, both absolute and relative, show a very irregular growth (figure 6 shows this effect).

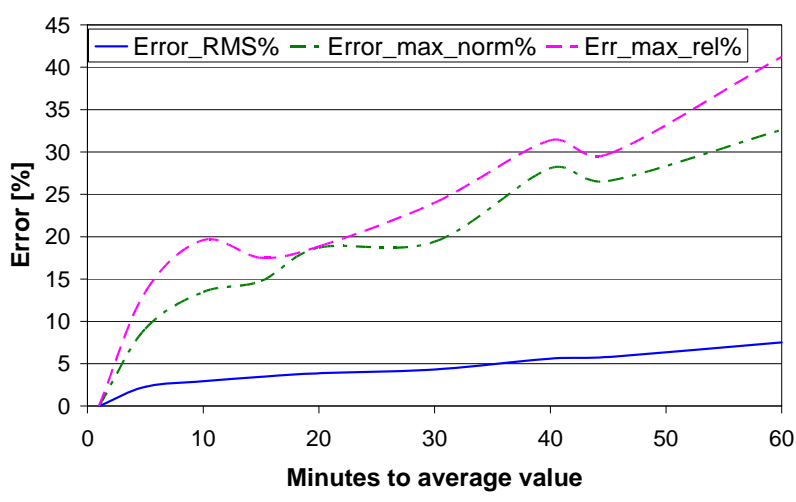

Fig. 6. Errors of a load curve as a function of minutes used to extend by means of a SH0 in a Tuesday of winter.

With regard to the interpolation using FFT, as shown in figure 4, the error decreases in an almost potential manner with the number of harmonics used for the interpolation. In addition, it might be noted that the moment that the maximum errors take place, in spite of not being a constant, there is a tendency toward a small number of points. Thus the maximum relative error is usually in off-peak hours. In contrast, the maximum standardized error usually occurs during peak hours.

If the two methods exposed are compared, interpolation SH0 and FFT, it can be noted that the method of interpolation FFT is more precise than that of holders, except for the relative error. In addition, this difference in errors grows (in relative terms) with the increase of the interpolation/ reconstruction period. Figure 7 shows the comparative evolution of errors for the interpolation methods exposed. From the observation of this figure, also it is clear that the interpolation/reconstruction based on FFT shows a much more stable and predictable evolution of errors than when the model based on retainers is in use. This lack of stability can lead to, occasionally for the analysis of the curve of a certain day, the algorithms based on holders showing minor relative 
or standardized errors (although only in some areas of the error curve).

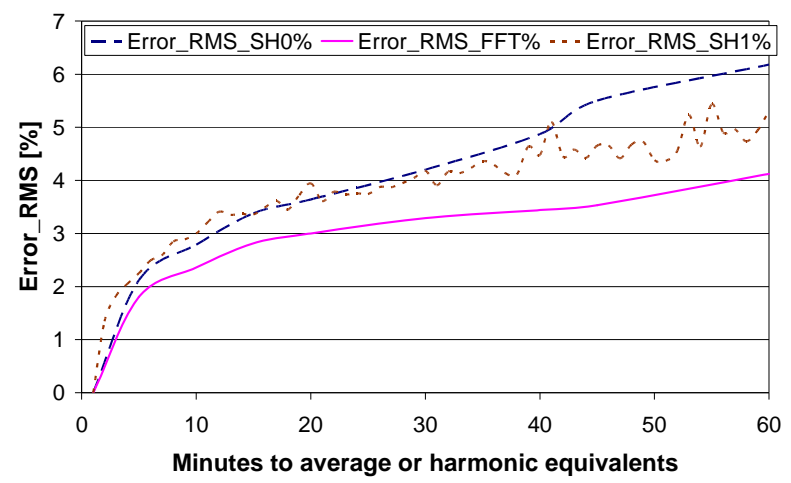

a)

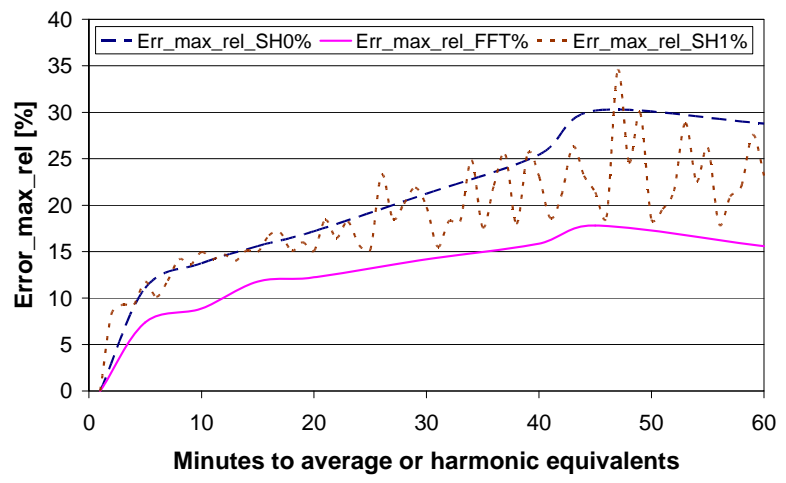

b)

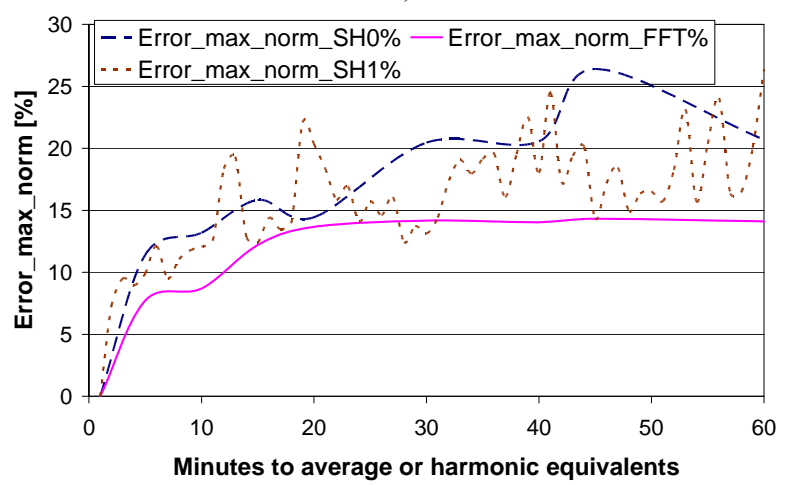

c)

Fig. 7. Comparison of errors using SH0 and FFT techniques.

However, when the period of interpolation is small (a few minutes), the errors committed by both algorithms of interpolation are very similar. So, on the basis of computational effort the use of models based on holders can be justified.

Studies based on wavelet decomposition are much more complex in its analysis, since there is a strong degree of dependence with the database used for decomposition. For this reason they are presented in independent publications, so that they can be adequately studied.

\section{Conclusions}

A comparison has been made of the behaviour of different methodologies for the interpolation and reconstruction of load curves in an urban LV substation.
We have analyzed the errors committed, in a hypothetical reconstruction (based on interpolation) of load curve. To do this the simulated curves have been contrasted with those obtained from an actual database.

It has been observed that the algorithms based on FFT are more accurate and reliable.

It has been found that, despite interpolation based on FFT being much more reliable, algorithms based on holders are valid when the period of reconstruction is small.

\section{Acknowledgement}

The authors would like to thank the "Ministerio de Ciencia e Innovación" of the Spanish Government for supporting this research under the Project ENE200914582-C02-02, as well as to thank to Iberdrola (La Rioja) for its collaboration in the measurements into the LV substation.

\section{References}

[1] N Cross, C. T. Gaunt, "Application of Rural Residential Hourly Load Curves in Energy Modelling”. IEEE PowerTech Conference Bologna 2003. 4 pages.

[2] V. Figueiredo, F. Rodrigues, Z. Vale, J. Borges, “A consumer characterization framework to support the definition of electricity supply contracts".

[3] C.S. Chen, M.S. Kang, J.C. Hwang, C.W. Huang, "Synthesis of power system load profiles by class load study". Elsevier Electrical Power and Energy Systems 22 (2000) 325330.

[4] G. J. Tsekouras, N. D. Hatziargyriou, Evangelos N. Dialynas," Two-Stage Pattern Recognition of Load Curves for Classification of Electricity Customers". IEEE Transactions on power systems, Vol. 22, NO. 3, AUGUST 2007. pg 1120-1128. [5] S. Valero, M. Ortiz, F. J. García, N. Encinas, A. Gabaldón, A. Molina, E. Gómez, "Characterization and Identification of Electrical Customers Through the Use of SelfOrganizing Maps and Daily Load Parameters", Power Systems Conference and Exposition, 2004. IEEE PES. 899 - 906 vol.2

[6] Amit Jain, B. Satish, "Short Term Load Forecasting by Clustering Technique based on Daily Average and Peak Loads", Power \& Energy Society General Meeting, 2009. PES '09. IEEE. pp. 1-7.

[7] D. Gerbec, S. Gašperič, F. Gubina, "Comparison of different classification methods for the consumers' load profile determination". Cired 17th International Conference on Electricity Distribution Barcelona, 12-15 May 2003. Paper 67

[8] J. Chen, W. Li, A.Lau, J. Cao, K. Wang "Automated Load Curve Data Cleansing in Power Systems". IEEE Transactions on smart grid, VOL. 1, NO. 2, september 2010. pp 213-221 